\title{
Metodologías activas y desarrollo de competencias en estudiantes universitarios con diferentes estilos de pensamiento
}

\author{
Active methodologies and competence development in higher education \\ students with different thinking styles
}

\author{
Trinidad García**, Olga Arias-Gundín*, Celestino Rodríguez**, \\ Raquel Fidalgo*, Patricia Robledo*
}

\author{
*Departamento de Psicología, Sociología y Filosofía. Facultad de Educación \\ Universidad de León \\ Campus de Vegazana, s/n, 24071, León. \\ ** Departamento de Psicología. Facultad de Psicología \\ Universidad de Oviedo \\ Plaza Feijoo, s/n, 33003, Oviedo. \\ garciatrinidad@uniovi.es oarig@unileon.es rodriguezcelestino@uniovi.es \\ rfidr@unileon.es probr@unileon.es
}

\begin{abstract}
Resumen
La implementación exitosa de metodologías activas para estimular el desarrollo de competencias en el contexto universitario requiere la consideración previa de aquellas características de los alumnos que pueden mediar en su manera de afrontar el aprendizaje, como son sus estilos de pensamiento. En este contexto se ha planteado el presente estudio el cual tiene como objetivos, en primer lugar, comparar el potencial diferencial de diferentes metodologías activas (expertos, aprendizaje basado en problemas, estudio de casos, estudio dirigido y estudio compartido) para estimular el desarrollo de competencias generales en los estudiantes universitarios y, en segundo lugar, analizar si el estilo de pensamiento de los alumnos predice el desarrollo de competencias en función de la metodología empleada. La muestra ha estado compuesta por 87 estudiantes de Grado de la Facultad de Educación de la Universidad de León (España), quienes cursaron varias asignaturas cuyas unidades temáticas fueron implementadas mediante las distintas metodologías activas objeto de análisis. Para valorar el estilo de pensamiento de los alumnos se empleó la adaptación española del Thinking Styles Inventory (Sternberg y Wagner, 1991). Para conocer el desarrollo de competencias percibido por los alumnos, éstos debieron responder, tras la implementación de cada metodología, al Cuestionario de Evaluación de las Competencias Transversales (Fidalgo, Arias y García, 2007). Los resultados indican, por un lado, que las cinco metodologías analizadas estimulan de manera similar el desarrollo de competencias en los universitarios, siendo las competencias instrumentales las más favorecidas en todos los métodos. Por otro lado, los resultados evidencian que los estilos de pensamiento de los alumnos y su edad actúan como variables predictoras del desarrollo competencial de manera diferente en relación a cada método. Por lo tanto, desde el punto de vista aplicado, se recomienda el uso combinado de diferentes metodologías activas para ajustarse a los perfiles diversos de la generalidad de los estudiantes y estimular así el óptimo desarrollo de las competencias transversales en todos ellos.
\end{abstract}

Palabras clave: Competencias generales, Estilos de pensamiento, Metodologías activas.

\begin{abstract}
The successful implementation of active methodologies to stimulate the development of competences in the university context requires the prior consideration of students characteristics that can mediate in their way of facing learning, such as their thinking styles. In this context, the present study aims to compare the potential of different active methodologies (experts, problem-based learning, case studies, guided study, and shared study) to stimulate the development of general competences in university students and, secondly, to analyze if students thinking styles predict the development of competences according to the methodology used. A sample of 87 undergraduate
\end{abstract}


students from the Faculty of Education, at the University of León (Spain), took part in the study, who attended several subjects whose content units were implemented through the different active methodologies under analysis. To evaluate students thinking style, the Spanish adaptation of the Thinking Styles Inventory (Sternberg \& Wagner, 1991) was used. In order to determine students competences development, they were requested to complete the Transversal Competences Assessment Questionnaire (Cuestionario de Evaluación de las Competencias Transversales) (Fidalgo, Arias y García, 2007), after the implementation of each active methodology. Results indicate, on the one hand, that the five methodologies analyzed stimulate in a similar way the development of competences in the students, being the instrumental competences the most favored by the different methods. On the other hand, it was also found that students thinking styles, and their age, act as predictors of competence development, but their effect was different in relation to each method. From a practical viewpoint, it is recommended the combined use of different active methodologies in order to fit the diverse students profiles and thus, stimulate the optimum development of transversal competences in all of them.

Key words: General competences, Thinking styles, Active methodologies.

\section{Introducción}

Las metodologías de enseñanza aprendizaje implementadas en la actualidad en las aulas universitarias, amparadas bajo los dictámenes del Espacio Europeo de Educación Superior, han de promover el desarrollo competencial de los universitarios y su capacidad de aprender a aprender de manera activa, autónoma y autorregulada, a lo largo de todo su proceso formativo y durante toda su vida profesional (Alcañiz et al., 2016; Arias y Fidalgo, 2013); por consiguiente, hoy en día los métodos didácticos que rigen las diferentes materias deben estar centrados más que nunca en el aprendiz y contribuir a que éste desarrolle, además de competencias profesionales específicas, competencias transversales de tipo instrumental, sistemático y personal que le faciliten su incorporación al mercado laboral (de Miguel, 2005; Villardón, 2015).

De este modo, parece lógico pensar que previamente a emprender un proceso de redefinición metodológica de una asignatura o materia es fundamental conocer y considerar las peculiaridades o características cognitivas concretas de los estudiantes, tales como sus enfoques de aprendizaje, su creatividad, su motivación o sus estilos de pensamiento, ya que éstas pueden determinar la eficacia de la metodología didáctica empleada (Albalate, Fageda, y Perdiguero, 2011; Bernardo, et al., 2011; Demirbas y Demirkan, 2007; Robledo et al., 2010). Pese a ello, el proceso de reconceptualización organizativa, estructural y funcional que impulsó hace ya casi dos décadas la Declaración de Bolonia (1999) parece inducir al uso en las aulas universitarias de métodos de enseñanza-aprendizaje de tipo activo ya que éstos, teóricamente, pretenden convertir al aprendiz en el agente protagonista de su proceso de aprendizaje, dotándole de una mayor autonomía y poder de decisión y actuación en el mismo. Sin embargo, cabe plantearse el interrogante de si todos los métodos activos son igualmente útiles para promover el desarrollo de competencias en todo el alumnado universitario o si las peculiaridades cognitivas individuales de cada alumnado pueden mediar en la eficacia del enfoque metodológico seguido.

En línea con este planteamiento, existen variedad de variables cognitivas internas del aprendiz que contribuyen a la explicación de la variabilidad en el rendimiento y desarrollo de los estudiantes universitarios (Demirbas y Demirkan, 2007, Robledo et al., 2010;). Entre estas variables cognitivas, alejándose progresivamente de la inteligencia como factor cognitivo único, en las últimas décadas han tomado cierto protagonismo los estilos de pensamiento. Éstos se definen como los procedimientos que utilizan las personas para activar los recursos de la inteligencia; serían, por lo tanto, los métodos que se emplean para organizar las cogniciones acerca del mundo; es decir, la manera predilecta de pensar de la persona (Sternberg, 1994, 1997, 1999; Stenberg y Grigorenko, 1995). Así, se entiende que el estilo de pensamiento de un estudiante se relaciona con su capacidad para dirigir su propia cognición y consecuentemente su propio proceso de aprendizaje. De modo que, por definición, los estilos de pensamiento muestran una intensa conexión con los parámetros autónomos de aprendizaje pretendidos 
por los actuales principios rectores de la enseñanza superior y por los métodos de tipo activo, siendo uno de los factores que mayor efecto modulador puede ejercer sobre la eficacia de los métodos de enseñanza aprendizaje y resultando por lo tanto de gran interés empírico-práctico.

La investigación empírica en el campo de los estilos de pensamiento ha demostrado a lo largo de los años la relación de esta variable con el rendimiento académico de los universitarios, bien de manera directa, bien por su correlación con otros factores cognitivos o emocionales (Bernardo, Zhang y Callueng, 2002; Bernardo et al., 2009; Cano-García y Hughes, 2000; Zhang, 2002, 2004); sin embargo, todavía hoy en día no hay un consenso firme acerca de qué estilos en particular son los que correlacionan positivamente con el rendimiento académico, siendo aún menor la evidencia cuando se operativiza el rendimiento en forma de competencia (Núñez et al., 2008; Zhang, 2004). Esto posiblemente se deba en parte a que dentro del propio abanico de estudiantes universitarios, sus estilos de pensamiento también muestran una gran variabilidad, existiendo trabajos que demuestran estilos muy dispares cuando se contrastan por ejemplo estudiantes de diferentes sexos, edades, tipos de universidades, especialidades, titulaciones, países, e incluso culturas (Allueva y Bueno, 2011; Bernardo, Fernández, et al., 2011; Bernardo, Núñez, et al., 2011; Fer, 2007; Grydley, 2007; Mogollón y Garrido, 2004; Robledo et al., 2010). Si a estas evidencias se unen aquellas que apoyan que los alumnos prefieren y se implican más en la realización de actividades de aprendizaje que son compatibles con sus estilos de pensamiento y que sólo algunos alumnos muestran combinaciones de estilos que les permiten ajustarse exitosamente a diferentes situaciones académicas, parece evidente que el estilo intelectual de los alumnos puede condicionar la eficacia de las metodologías activas y su potencial para promover el desarrollo de competencias (González, 2002; González-Pienda, Roces, Bernardo, y García, 2002; Martínez y Crespo, 2008).

\section{Objetivos}

Sin embargo, pese a los avances producidos en este campo de conocimiento, a partir de la revisión empírica realizada, no se han localizado trabajos en el contexto español en los que se analicen de manera conjunta las relaciones existentes entre estas tres variables: metodología activa empleada por los docentes, rendimiento competencial logrado en los alumnos y estilo de pensamiento predilecto de ellos. Es necesario por lo tanto profundizar en este ámbito de estudio con el objeto de encontrar evidencias que permitan orientar al profesorado a la hora de tomar decisiones acerca del proceder metodológico que asumir en sus materias considerando el estilo de pensamiento de sus alumnos, especialmente si se persiguen la mejora de la calidad del sistema universitario, la excelencia en la docencia y el éxito académico de todos los estudiantes, al hilo de los objetivos de la iniciativa de la Estrategia Universidad 2015.

Con este propósito se plantea la presente investigación, la cual tiene un doble objetivo, comparativo y predictivo. Así, en primer lugar, se pretende conocer si existen diferencias en el desarrollo de competencias (instrumentales, sistémicas y personales) de los alumnos en función de la metodología de enseñanza-aprendizaje implementada (expertos, aprendizaje basado en problemas, estudio de casos, estudio dirigido y estudio compartido). Es decir, comparar el potencial diferencial de cada metodología para optimizar el desarrollo de competencias en los alumnos. Se trata de métodos de tipo activo, por lo que presumiblemente demandarán la activación de recursos similares en los alumnos, esperándose que el nivel de desarrollo competencial que promuevan todos ellos sea similar. En segundo lugar, se quiere determinar si el estilo de pensamiento de los estudiantes predice el desarrollo de competencias en función del tipo de metodología empleado. Para ello, este estudio se centrará en aquellas competencias que muestren estar más desarrolladas por parte de los estudiantes. Se pretende saber si unas metodologías son más adecuadas-eficaces que otras para favorecer la asimilación de dichas competencias en los alumnos según el estilo de pensamiento de éstos. Los estilos intelectuales se examinan en relación a cinco dimensiones, en torno a las que se agrupan los trece estilos de pensamiento descritos en la teoría de la mente (Sternberg, 1988): Funciones: legislativo, judicial y ejecutivo; Formas: monárquico, jerárquico, oligárquico y anárquico; Niveles: global y local; Ámbito: interno y externo; Tendencias: conservador y 
liberal. En este caso, se espera que aquellos estilos de pensamiento que deriven en la puesta en marcha de actividades o procesos relacionados o compatibles con las demandas de cada metodología contribuyan a fomentar un mayor desarrollo de competencias; ya que los alumnos se implican más en las tareas que son compatibles con sus estilos de autogobierno (González-Pienda, Roces, Bernardo, y García, 2002).

\section{Método}

\subsection{Participantes}

La muestra del estudio la formaron 87 estudiantes universitarios matriculados en segundo y tercer curso del Grado de Educación Especial $(\mathrm{n}=24$, tercer curso), Educación Física $(\mathrm{n}=25$, segundo curso) y Educación Primaria ( $\mathrm{n}=38$, segundo curso) de la Facultad de Educación de la Universidad de León (España). Sus edades estaban comprendidas entre los 19 y los 33 años, siendo la media de edad de 21,12 años. El 66,2\% de la muestra eran mujeres mientras que el 33,8\% eran hombres.

El tipo de muestreo empleado para la selección de la muestra ha sido intencional o de conveniencia ya que se han seleccionado directamente a los alumnos matriculados en las materias impartidas por los profesores colaboradores en el estudio. De este modo todos los alumnos universitarios cursaron una o varias asignaturas troncales propias de su mención, en las que uno o varios temas fueron implementadas por parte del profesorado empleando para ello el uso de las siguientes metodologías activas: método de expertos, aprendizaje basado en problemas, estudio de casos, estudio dirigido y estudio compartido. En la Tabla 1 se recoge la distribución de la muestra por mención, metodología implementada y tema concreto.

\begin{tabular}{|c|c|c|c|c|c|c|c|c|c|c|}
\hline \multirow{3}{*}{ Especialidad } & \multicolumn{10}{|c|}{ Metodología } \\
\hline & \multicolumn{2}{|c|}{ EXP } & \multicolumn{2}{|c|}{ ABP } & \multicolumn{2}{|c|}{ CASO } & \multicolumn{2}{|c|}{ EDI } & \multicolumn{2}{|c|}{ ECO } \\
\hline & Tema & $\begin{array}{c}N^{o} \\
\text { alumnos }\end{array}$ & Tema & $\begin{array}{c}N^{o} \\
\text { alumnos }\end{array}$ & Tema & $\begin{array}{c}N^{o} \\
\text { alumnos }\end{array}$ & Tema & $\begin{array}{c}N^{o} \\
\text { alumnos }\end{array}$ & Tema & $N^{o}$ alumnos \\
\hline Ed. Especial & Legislación & 23 & $\begin{array}{l}\text { Parálisis } \\
\text { cerebral }\end{array}$ & 24 & Espina bífida & 24 & Miopatías & 24 & $\begin{array}{c}\text { Desarrollos } \\
\text { generales }\end{array}$ & 24 \\
\hline \multirow{2}{*}{ Ed. Física } & $\begin{array}{c}\text { Desarrollo } \\
\text { motor ciclo } \\
\text { vital }\end{array}$ & 24 & $\begin{array}{c}\text { Desarrollo } \\
\text { primera } \\
\text { infancia }\end{array}$ & 24 & $\begin{array}{c}\text { Desarrollo } \\
\text { etapa infantil } \\
\text { Aprendizajes }\end{array}$ & $\begin{array}{l}22 \\
25\end{array}$ & - & - & - & - \\
\hline & $\begin{array}{c}\text { Deficiencias } \\
\text { sensoriales y } \\
\text { fisicas }\end{array}$ & 19 & TDAH & 21 & - & - & Sobredotación & 21 & $\begin{array}{l}\text { Dificultades } \\
\text { aprendizaje }\end{array}$ & 20 \\
\hline Ed. Primaria & $\begin{array}{c}\text { Deficiencias } \\
\text { sensoriales y } \\
\text { físicas }\end{array}$ & 38 & TDAH & 38 & - & - & Sobredotación & 38 & $\begin{array}{l}\text { Dificultades } \\
\text { aprendizaje }\end{array}$ & 37 \\
\hline $\begin{array}{l}\text { Total } \\
\text { metodología }\end{array}$ & & 104 & & 107 & & 71 & & 83 & & 81 \\
\hline
\end{tabular}

Tabla 1: Distribución de la muestra de alumnos participantes en el estudio

\subsection{Instrumentos}

\section{Cuestionario de Estilos de Pensamiento}

Para valorar los estilos de pensamiento de los estudiantes que participaron en el estudio se utilizó la adaptación realizada por González-Pienda y colaboradores (1997) del Thinking Styles Inventory (MSG, Sternberg y Wagner, 1991). Este cuestionario, estandarizado y validado empíricamente en castellano y ampliamente utilizado a nivel nacional e internacional, consta de 104 ítems divididos en trece escalas, con ocho ítems en cada una de ellas. Para cada ítem el estudiante debía responder mediante una escala tipo likert de 1 a 7 , donde el 1 indicaba ningún acuerdo con el ítem y el 7 representaba el máximo acuerdo. Las trece escalas corresponden con los trece estilos de pensamiento descritos en la Teoría Del Autogobierno Mental (Sternberg, 1988) y se agrupan en cinco dimensiones: i) Funciones: legislativo, 
judicial y ejecutivo; ii) Formas: monárquico, jerárquico, oligárquico y anárquico; iii) Niveles: global y local; iv) Ámbito: interno y externo; v) Tendencias: conservador y liberal.

\section{Cuestionario de Evaluación de las Competencias Transversales}

Por su parte, para conocer, desde la perspectiva del propio estudiante, el grado en el que las metodologías de enseñanza-aprendizaje potencian o facilitan la adquisición de competencias generales, se utilizó el Cuestionario de Evaluación de las Competencias Transversales (Fidalgo, Arias y García, 2007). Este cuestionario valora la percepción del alumno en torno al desarrollo o adquisición personal de un total de 23 competencias, ocho de tipo instrumental, ocho de carácter sistémico y las siete restantes de índole personal. En la Tabla 2 se recogen las competencias concretas valoradas por el instrumento.

\begin{tabular}{|c|c|}
\hline Tipo & Competencia \\
\hline Instrumental & $\begin{array}{l}\text { - Capacidad de análisis y síntesis } \\
\text { - Capacidad de organización y planificación } \\
\text { - Capacidad de comunicación oral y escrita en lengua nativa } \\
\text { - Conocimiento de una lengua extranjera } \\
\text { - Conocimientos de informática relativos al ámbito de estudio } \\
\text { - Capacidad de gestión de la información } \\
\text { - Ser capaz de tomar decisiones }\end{array}$ \\
\hline Personal & $\begin{array}{l}\text { - Capacidad para trabajar en equipo y colaborar eficazmente con otras personas } \\
\text { - Capacidad para trabajar en equipos de carácter interdisciplinar } \\
\text { - Capacidad para trabajar en un contexto internacional } \\
\text { - } \text { Habilidades en las relaciones interpersonales } \\
\text { - Reconocimiento a la diversidad y la multiculturalidad } \\
\text { - Compromiento crítico } \\
\text { Comótico }\end{array}$ \\
\hline Sistémica & $\begin{array}{l}\text { - Capacidad para desarrollar y mantener actualizadas las propias competencias, destrezas y } \\
\text { - Conocimientos según los estándares de la profesión } \\
\text { - Capacidad para adaptarse a nuevas situaciones } \\
\text { - Capacidad para pensar de forma creativa y desarrollar nuevas ideas y conceptos } \\
\text { - Conocimiento de otras culturas y costumbres } \\
\text { - Iniciativa y espíritu emprendedor } \\
\text { - Mostrar interés por la calidad de la propia actuación y saber desarrollar sistemas para } \\
\text { garantizar la calidad de los propios servicios } \\
\text { - Sensibilidad hacia temas ambientales }\end{array}$ \\
\hline
\end{tabular}

Tabla 2: Competencias valoradas por el Cuestionario de Evaluación de las Competencias Transversales

El cuestionario está constituido por un total de 69 ítems agrupados de tres en tres, de manera que cada conjunto de tres ítems operativiza cada una de las 23 competencias valoradas. De este modo, la escala ofrece una primera medida, de carácter individual para cada competencia, que se obtiene sumando la respuesta dada por el estudiante a los tres ítems que la conforman; pero, además, facilita la obtención de un subtotal específico para cada tipología concreta de competencias, diferenciando entre competencias instrumentales, sistémicas y personales. Finalmente, el cuestionario ofrece una última medida de la adquisición total de competencias, resultante de la suma de los tres subtotales anteriores.

Cada ítem de respuesta cerrada está enunciado en la forma "En qué medida esta metodología ha favorecido mi capacidad para..."; oscilando la escala de respuesta de 1 al 9, siendo 1 el mínimo grado de desarrollo y 9 el máximo. Estudios experimentales previos han corroborado una adecuada validez de 
contenido y una alta fiabilidad del cuestionario (Fidalgo, Arias y García, 2007), obteniéndose, para el contexto específico de esta investigación, un alfa de Cronbach de .972.

\subsection{Descripción de las metodologías activas}

Cada una de las metodologías activas analizas en este estudio se caracteriza por tener una secuencia de aplicación concreta que le confiere entidad propia y en la que, tanto el rol del profesor y del alumno, como las tareas o actividades que se deben desarrollar para lograr los objetivos de aprendizaje pretendidos, se definen claramente. Por lo tanto, a continuación, se muestra una síntesis descriptiva de las distintas metodologías, a fin de clarificar el modo en el que el profesorado implicado en el estudio ha llevado a cabo la implementación de las mismas.

Comenzando en primer lugar por el estudio de caso, este método comienza con la actuación didáctica del profesor quien, tras explicar los fundamentos teóricos de la unidad temática y realizar una presentación y descripción del caso a abordar, de manera oral y por escrito. Posteriormente, de manera grupal se clarifican los aspectos básicos a analizar, identificando los puntos más importantes del caso. A continuación, se relacionan estos aspectos con los fundamentos teóricos que ayuden a la comprensión y resolución del caso. Seguidamente los alumnos plantean posibles alternativas de solución y buscan los argumentos que defiendan las mejores ideas, para seleccionarlas. En este punto, se toma decisiones de equipo, negociando y concretando la propuesta estimada por el grupo como más relevante; ésta se aplica al caso desarrollando una estrategia que permita llevar a la práctica dicha solución y sus posibles consecuencias. Finalmente se pide a los alumnos que elaboren un informe escrito en el que exprese la solución considerada por el grupo como idónea.

En cuanto al método del aprendizaje basado en problemas, éste comienza con la presentación del problema por parte del profesor mediante la lectura en grupo e individual del mismo, enumerando los puntos de interés del mismo e identificando los que se consideren importantes, desconocidos o no comprendidos. Seguidamente el problema es analizado y discutido por los estudiantes utilizando sus conocimientos previos. En este caso se les pide que elaboren un mapa conceptual donde deben figurar todos los temas ligados al problema. Seguidamente, se lleva a cabo la distribución de las tareas a realizar entre los componentes del equipo y se procede a realizar el trabajo autónomo de los contenidos. En este punto se pide a los alumnos que redacten un informe resumen de su trabajo destinado a sus compañeros, para que todos tengan la información completa. De nuevo en grupo se discute y enfoca el problema inicial, en base a los nuevos conocimientos adquiridos y, en caso de necesidad se modifica el mapa conceptual provisional. Llegados a este punto los estudiantes ya proponen la resolución al problema planteado y elaboran un informe final.

Por su parte el método de expertos sigue la siguiente secuencia de implementación. En primer lugar, la clase es dividida en equipos y el tema general de estudio, en tantas partes o sub-temas como miembros forman los equipos. El profesor facilita a los alumnos materiales de formación básica para formar a los expertos en cada sub-tema. Cada miembro de los equipos elige o se le asigna un sub-tema a aprender. Es necesario que se convierta en experto de su tema, de manera que tiene que llevar a cabo un trabajo formativo individual. Una vez formado en su sub-tema cada experto se reunirá en un seminario de expertos con los expertos de su sub-tema del resto de los grupos. Cada experto, una vez formado y comprobado su buen nivel, se reunirá con su equipo inicial en un seminario de formación. Él será el responsable de la formación en su sub-tema del resto del grupo. Finalmente se pide a los alumnos que realicen un trabajo grupal y lo defiendan.

En cuarto lugar, el estudio compartido parte de la exposición de contenidos por parte del profesor, y la escucha activa de los alumnos quienes, a continuación, deben resolver actividades ejemplo. Estas actividades son corregidas seguidamente. En este caso, el proceso de análisis y resolución de las actividades, se realiza inicialmente en pequeños grupos de trabajo, para posteriormente realizarse una puesta en gran grupo en la que se corrigen los posibles errores y se reflexiona sobre los resultados. Por 
último, para consolidar el aprendizaje se realizan tareas de repaso, de las cuales se entrega un informe final al profesorado.

Finalmente, en el método de estudio dirigido el profesor inicialmente elabora una guía de estudio que presenta a los alumnos, resolviendo sus dudas. El alumnado de forma individual prepara el tema con el apoyo de la guía, consultando para ello las fuentes documentales señaladas en la misma y realizando el plan de actividades propuesto. Además, deben monitorizar de manera independiente su proceso de aprendizaje para lo que se les recomienda que estudien los pasos a seguir en el desarrollo del tema y elaboren un cronograma de trabajo. Durante todo el proceso pueden consultar dudas tanto en la bibliografía propuesta como al propio profesor.

\subsection{Diseño y análisis estadístico}

Se llevó a cabo un diseño cuasi-experimental de un grupo solo postest. Con el fin de cumplir los diferentes objetivos planteados, el análisis de datos se realizó en diferentes pasos. En primer lugar, se analizó la distribución de las variables a estudiar, con el fin de determinar la conveniencia o no de realizar análisis paramétricos. Se prestó especial atención a los valores de asimetría, curtosis, y a la prueba de Kolmogorov-Smirnov. En la Tabla 3 se muestran los resultados de estos análisis, los cuales muestran que, tras unos ajustes en el tamaño muestral, todas las variables excepto la edad cumplen con los criterios de normalidad. En segundo lugar, con el objetivo de determinar las diferencias intra-sujeto en el grado de desarrollo de las diferentes competencias bajo cada método de enseñanza-aprendizaje, se realizaron análisis de medidas repetidas, donde se compararon las percepciones de los estudiantes en el desarrollo de las tres competencias. Se realizaron análisis separados para cada una de las 5 metodologías empleadas (Tabla 4). Dado que se trata de 3 tipos de competencias, se estableció en un estadístico para determinar entre qué competencias se encuentran las diferencias. Se empleó la prueba de Bonferroni, que también está disponible para medidas repetidas. Una vez determinado el tipo de competencias más desarrolladas por los estudiantes, se pasan a analizar las variables que mejor predicen el desarrollo de dichas competencias. Como posibles variables predictoras se incluyen las trece variables de estilos de pensamiento y la edad. La variable dependiente o a predecir ha sido las competencias instrumentales, bajo cada una de las metodologías de enseñanza-aprendizaje empleadas. Se realizan, por tanto, cinco análisis de regresión, uno para cada metodología. Se emplea el método backward o hacia atrás como método de selección de variables, ya que hay una gran cantidad de variables predictoras y en algunos de los métodos una muestra pequeña, además de que se carece de un cuerpo de teoría y evidencias empíricas que permitan tener una hipótesis clara acerca de qué predictores incluir en el modelo. Se incluyó la edad como posible variable predictora dado que ha mostrado una gran variabilidad en los análisis descriptivos. En la Tabla 5 se presentan los resultados del análisis.

\subsection{Procedimiento}

El estudio se llevó a cabo a lo largo de todo un curso escolar en el que los profesores colaboradores implicados en el desarrollo del trabajo, concretamente dos profesores Titulares de Universidad del área de Psicología Evolutiva y de la Educación, al comienzo de cada una de las asignaturas de las que eran responsables administraron a sus alumnos el cuestionario de estilos de pensamiento.

Posteriormente se procedió al desarrollo propiamente dicho de las materias. Inicialmente, se llevó a cabo un análisis y entrenamiento en profundidad por los profesores participantes de los diferentes métodos de enseñanza-aprendizaje a aplicar, prestando especial atención, además de a las evidencias empíricas que demostraban su eficacia, al proceso de implementación que se debía seguir en cada uno de ellos. Seguidamente, los profesores implicados en el estudio analizaron la situación concreta de cada asignatura, analizando su temario específico y determinando qué método concreto, de los cinco a estudiar, se utilizaría para la implementación de cada unidad temática específica. De este modo, 
diferentes temas de las diversas asignaturas fueron desarrollados mediante alguna de las modalidades metodológicas objeto de estudio (ver para más detalle Tabla 1).

A continuación, en base a este planteamiento organizativo metodológico, se comenzó con la impartición propiamente dicha de las materias, implementando para ello el proceso de enseñanza-aprendizaje de cada unidad temática según la secuencia o procedimiento que dicta la metodología activa previamente decidida para abordar ese tema. Cada vez que finalizaba la aplicación de alguno de los temas impartidos en base a algún método de los comparados, los estudiantes respondían el Cuestionario de Evaluación de las Competencias Transversales, para reflejar su percepción inmediata acerca de cómo la metodología didáctica utilizada había contribuido al desarrollo de las tres tipologías de competencias estudiadas.

Concluido el trabajo de campo y recogidos todos los cuestionarios se procedió a la informatización y codificación de los datos en una matriz de datos Excel, la cual se transformó en una matriz de Statistical Package for the Social Sciences (SPSS), versión 20, para la realización de los análisis estadísticos oportunos.

\section{Resultados}

Inicialmente se ha analizado la distribución de las variables a estudiar, atendiendo a los valores de asimetría, curtosis, y a la prueba de Kolmogorov-Smirnov. En este caso se ha detectado que varias variables no cumplieron el criterio de normalidad, por lo que se analizaron los outliers y se eliminaron aquellos sujetos con puntuaciones extremas. Se repitieron los análisis de la distribución y el test de normalidad una vez reducida la muestra. Los resultados indicaron entonces que solamente la variable edad se desvía de la normalidad. Las demás variables siguen una distribución normal (ver para más detalle Tabla 3). No obstante, hay que tener en cuenta que en cada uno de los métodos se cuenta con un tamaño muestral diferente, lo cual, unido a la pérdida de muestra referida anteriormente, restringe parcialmente el análisis de los datos.

\begin{tabular}{|c|c|c|c|c|c|c|c|}
\hline & MAX & MIN & $M$ & $D T$ & ASIM & CURT & $K-S(p)$ \\
\hline EDAD & 19 & 33 & 21.12 & 2.373 & 2.583 & 9.436 & $\begin{array}{l}2.014 \\
(.001)\end{array}$ \\
\hline \multicolumn{8}{|c|}{ EXP } \\
\hline $\begin{array}{l}\text { TOTAL } \\
\text { INSTRUMENTALES }\end{array}$ & 77 & 180 & 139.83 & 25.106 & -.674 & .554 & $\begin{array}{c}.695 \\
(.720)\end{array}$ \\
\hline TOTAL SISTÉMICAS & 37 & 179 & 128.52 & 34.784 & -.814 & .796 & $\begin{array}{c}.497 \\
(.966)\end{array}$ \\
\hline TOTAL PERSONALES & 71 & 167 & 122.13 & 22.660 & -.187 & .131 & $\begin{array}{c}.514 \\
(.954) \\
\end{array}$ \\
\hline \multicolumn{8}{|c|}{ ECO } \\
\hline $\begin{array}{l}\text { TOTAL } \\
\text { INSTRUMENTALES }\end{array}$ & 46 & 187 & 132.53 & 28.682 & -.566 & .117 & $\begin{array}{c}.730 \\
(.660)\end{array}$ \\
\hline TOTAL SISTÉMICAS & 43 & 190 & 127.51 & 29.374 & -.399 & .151 & $\begin{array}{c}.492 \\
(.969)\end{array}$ \\
\hline TOTAL PERSONALES & 43 & 165 & 109.45 & 26.873 & -.392 & -.246 & $\begin{array}{c}.546 \\
(.926) \\
\end{array}$ \\
\hline \multicolumn{8}{|c|}{ EDI } \\
\hline $\begin{array}{l}\text { TOTAL } \\
\text { INSTRUMENTALES }\end{array}$ & 48 & 187 & 141.99 & 26.166 & -.901 & 1.635 & $\begin{array}{c}.906 \\
(.384)\end{array}$ \\
\hline TOTAL SISTÉMICAS & 50 & 188 & 136.51 & 29.341 & -.601 & .141 & $\begin{array}{c}.804 \\
(.538)\end{array}$ \\
\hline TOTAL PERSONALES & 40 & 169 & 115.41 & 28.335 & -.561 & .231 & $\begin{array}{c}.521 \\
(.949)\end{array}$ \\
\hline
\end{tabular}


RIDU Revista d'Innovacio Docent Universitaria $9(2017)$ (66-80

\begin{tabular}{|c|c|c|c|c|c|c|c|}
\hline \multicolumn{8}{|c|}{$\mathrm{ABP}$} \\
\hline $\begin{array}{l}\text { TOTAL } \\
\text { INSTRUMENTALES }\end{array}$ & 86 & 192 & 138.31 & 23.413 & -.042 & .113 & $\begin{array}{c}.479 \\
(.976)\end{array}$ \\
\hline TOTAL SISTÉMICAS & 88 & 207 & 132.82 & 23.903 & .645 & 1.274 & $\begin{array}{c}.690 \\
(.728)\end{array}$ \\
\hline TOTAL PERSONALES & 77 & 171 & 121.49 & 21.415 & .190 & .518 & $\begin{array}{c}.830 \\
(.496) \\
\end{array}$ \\
\hline \multicolumn{8}{|c|}{ CASO } \\
\hline $\begin{array}{l}\text { TOTAL } \\
\text { INSTRUMENTALES }\end{array}$ & 73 & 182 & 131.43 & 24.794 & -.337 & -.051 & $\begin{array}{c}.418 \\
(.995)\end{array}$ \\
\hline TOTAL SISTÉMICAS & 61 & 187 & 124.02 & 26.571 & -.175 & -.007 & $\begin{array}{c}.509 \\
(.958)\end{array}$ \\
\hline TOTAL PERSONALES & 62 & 149 & 111.31 & 22.304 & -.277 & -.506 & $\begin{array}{c}.470 \\
(.980)\end{array}$ \\
\hline \multicolumn{8}{|c|}{ ESTILOS } \\
\hline LEGISLATIVO & 2.87 & 6.500 & 4.84 & .744 & -.465 & .293 & $\begin{array}{c}.887 \\
(.410)\end{array}$ \\
\hline EJECUTIVO & 3.25 & 6.13 & 4.75 & .660 & -.049 & -.413 & $\begin{array}{l}.740 \\
(.645)\end{array}$ \\
\hline JUDICIAL & 2.88 & 5.88 & 4.57 & .630 & -.120 & -.336 & $\begin{array}{c}.879 \\
(.423)\end{array}$ \\
\hline GLOBAL & 2.75 & 11.625 & 4.59 & 1.043 & 3.379 & 21.869 & $\begin{array}{l}1.301 \\
(.068)\end{array}$ \\
\hline LOCAL & 2.50 & 6.000 & 4.24 & .652 & -.143 & .209 & $\begin{array}{l}.737 \\
(.650)\end{array}$ \\
\hline LIBERAL & 2 & 7 & 4.52 & .894 & .026 & -.151 & $\begin{array}{c}.612 \\
(.848)\end{array}$ \\
\hline CONSERVADOR & 2.00 & 6.00 & 4.16 & .830 & -.228 & -.355 & $\begin{array}{c}.872 \\
(.432)\end{array}$ \\
\hline JERÁRQUICO & 2.6 & 6.5 & 4.83 & .831 & -.380 & -.212 & $\begin{array}{l}1.005 \\
(.265)\end{array}$ \\
\hline MONÁRQUICO & 2.12 & 5.75 & 4.37 & .691 & -.284 & .370 & $\begin{array}{c}.764 \\
(.603)\end{array}$ \\
\hline OLIGÁRQUICO & 2.5 & 6.5 & 4.72 & .838 & -.207 & -.052 & $\begin{array}{c}.725 \\
(.6709\end{array}$ \\
\hline ANÁRQUICO & 2.75 & 6.00 & 4.25 & .628 & .250 & .295 & $\begin{array}{c}.648 \\
(.795)\end{array}$ \\
\hline INTERNO & 2 & 6 & 4.23 & .721 & -.152 & -.474 & $\begin{array}{c}.776 \\
(.584)\end{array}$ \\
\hline EXTERNO & 2.75 & 6.88 & 5.05 & .823 & -.293 & .112 & $\begin{array}{c}.940 \\
(.340)\end{array}$ \\
\hline
\end{tabular}

Nota: EXP = método de experto; ECO = estudio compartido; EDI = estudio dirigido; ABP = aprendizaje basado en problemas; CASO = estudio de caso.

Tabla 3: Estadísticos descriptivos de las variables de estudio y ajuste a la distribución normal

\subsection{Desarrollo de los diferentes tipos de Competencias en función de las Metodologías Activas empleadas}

En cuanto al análisis de las competencias más desarrolladas dentro de cada metodología, y teniendo en cuenta las diferentes muestras en las metodologías, se realizaron análisis de medidas repetidas. Concretamente, se compararon, dentro de cada metodología, las medias de los sujetos en competencias instrumentales, sistemáticas y personales.

Los resultados de los análisis de medidas repetidas, recogidos en la Tabla 4, indicaron que las competencias instrumentales son las más desarrolladas en todas las metodologías. Los tamaños del efecto de las diferencias son elevados, con una eta cuadrado en torno a .70 . 


\begin{tabular}{|c|c|c|c|c|c|c|c|c|}
\hline \multirow[b]{2}{*}{ MÉTODO } & \multirow{2}{*}{$\frac{\text { INST }}{M(D T)}$} & \multirow{2}{*}{$\begin{array}{c}\text { SIST } \\
M(D T)\end{array}$} & \multirow{2}{*}{$\begin{array}{c}\text { PERS } \\
M(D T)\end{array}$} & \multicolumn{5}{|c|}{ Diferencias } \\
\hline & & & & $F$ & g.l & $p$ & $\eta p^{2}$ & Grupos \\
\hline EXP & $\begin{array}{c}139.83 \\
(25.106)\end{array}$ & $\begin{array}{c}128.52 \\
(34.784)\end{array}$ & $\begin{array}{c}122.13 \\
(22.660)\end{array}$ & 47.648 & 2,21 & $<.001$ & .819 & I-S, I-P \\
\hline $\mathrm{ECO}$ & $\begin{array}{c}132.53 \\
(28.682)\end{array}$ & $\begin{array}{c}127.51 \\
(29.374)\end{array}$ & $\begin{array}{c}109.45 \\
(26.873)\end{array}$ & 119.989 & 2,75 & $<.001$ & .762 & Todos \\
\hline EDI & $\begin{array}{c}141.99 \\
(26.116)\end{array}$ & $\begin{array}{c}136.51 \\
(29.341)\end{array}$ & $\begin{array}{c}115.41 \\
(28.335)\end{array}$ & 126.466 & 2,78 & $<.001$ & .764 & Todos \\
\hline ABP & $\begin{array}{c}138.31 \\
(23.413)\end{array}$ & $\begin{array}{c}132.82 \\
(23.903)\end{array}$ & $\begin{array}{c}121.49 \\
(21.415)\end{array}$ & 52.200 & 2,43 & $<.001$ & .708 & Todos \\
\hline CASO & $\begin{array}{c}131.43 \\
(24.794)\end{array}$ & $\begin{array}{c}124.02 \\
(26.571)\end{array}$ & $\begin{array}{c}111.31 \\
(22.304)\end{array}$ & 39.192 & 2,40 & $<.001$ & .662 & Todos \\
\hline
\end{tabular}

Nota: $\mathrm{EXP}=$ método de experto; $\mathrm{ECO}$ = estudio compartido; $\mathrm{EDI}$ = estudio dirigido; $\mathrm{ABP}$ = aprendizaje basado en problemas; $\mathrm{CASO}=$ estudio de caso.

Tabla 4: Resultados de los análisis de medidas repetidas

Así pues, analizando la Tabla 4 se evidencia que generalmente, hay diferencias estadísticamente significativas entre las competencias en todas las metodologías; si bien en todos los casos el tipo de competencias más desarrolladas han sido las de tipo instrumental, razón por la cual pasamos a analizar qué variables predicen que el alumnado desarrollo específicamente estas competencias.

\subsection{Resultados del análisis de regresión}

Puesto que han sido las competencias instrumentales las que han demostrado estar más desarrolladas en todas las metodologías, cabe preguntarse, al hilo del segundo objetivo de este estudio qué variables relativas a los estilos de pensamiento predicen el desarrollo de estas competencias en cada una de las metodologías.

En la Tabla 5 se presentan los resultados de los análisis de regresión, los cuales se realizaron separadamente para cada metodología de enseñanza-aprendizaje. Como posibles variables predictoras, se analizan la edad y los treces estilos de pensamiento. La variable dependiente son las puntuaciones de los estudiantes en la escala que mide competencias instrumentales.

\begin{tabular}{|c|c|c|c|c|c|c|c|c|c|c|c|c|}
\hline & \multicolumn{3}{|c|}{ EXP } & \multicolumn{3}{|c|}{ EDI } & \multicolumn{3}{|c|}{ ABP } & \multicolumn{3}{|c|}{ CASO } \\
\hline & Variable & Beta & $t(p)$ & Variable & Beta & $t(p)$ & Variable & Beta & $t(p)$ & Variable & Beta & $t(p)$ \\
\hline \multirow{5}{*}{$\begin{array}{l}\text { Variables } \\
\text { en la } \\
\text { ecuación }\end{array}$} & EDAD & .466 & $\begin{array}{c}3.134 \\
(\mathrm{p}=.006)\end{array}$ & \multirow[t]{4}{*}{ EJEC } & \multirow[t]{4}{*}{4.56} & \multirow[t]{4}{*}{$\begin{array}{c}3.509 \\
(\mathrm{p}=.001)\end{array}$} & EDAD & .304 & \multirow{4}{*}{$\begin{array}{c}2.079 \\
(\mathrm{p}=.044) \\
2.298 \\
(\mathrm{p}=.013)\end{array}$} & EDAD & .403 & \multirow{5}{*}{$\begin{array}{c}3.100 \\
(\mathrm{p}=.004) \\
4.403 \\
(\mathrm{p}<.001) \\
-2.415 \\
(\mathrm{p}=.021)\end{array}$} \\
\hline & INT & .445 & $\begin{array}{c}2.651 \\
(\mathrm{p}=.016)\end{array}$ & & & & \multirow[t]{4}{*}{ MON } & \multirow[t]{3}{*}{.379} & & MON & .664 & \\
\hline & MON & .619 & $\begin{array}{c}23.411 \\
(\mathrm{p}=.003)\end{array}$ & & & & & & & \multirow[t]{2}{*}{ EXT } & \multirow[t]{2}{*}{-.329} & \\
\hline & JUD & -.809 & $\begin{array}{c}-4.219 \\
(\mathrm{p}=.001)\end{array}$ & & & & & & & & & \\
\hline & 52.3 & 12.3 & 13.9 & 40.9 & & & & & & & & \\
\hline $\mathrm{R}^{2}$ & \multicolumn{3}{|c|}{$\begin{array}{c}\mathrm{F} 4,22=7.243 \\
(\mathrm{p}=.001)\end{array}$} & \multicolumn{3}{|c|}{$\begin{array}{c}\mathrm{F} 5,69=3.436 \\
(\mathrm{p}<.001)\end{array}$} & \multicolumn{3}{|c|}{$\begin{array}{c}\mathrm{F} 2,41=4.471 \\
(\mathrm{p}=.018)\end{array}$} & \multicolumn{3}{|c|}{$\begin{array}{c}\mathrm{F} 4,36=7.930 \\
(\mathrm{p}<.001)\end{array}$} \\
\hline F & \multicolumn{3}{|c|}{$\begin{array}{c}\mathrm{F} 4,22=7.243 \\
(\mathrm{p}=.001)\end{array}$} & \multicolumn{3}{|c|}{$\begin{array}{c}\mathrm{F} 5,69=3.436 \\
(\mathrm{p}<.001)\end{array}$} & \multicolumn{3}{|c|}{$\begin{array}{c}\mathrm{F} 2,41=4.471 \\
(\mathrm{p}=.018)\end{array}$} & \multicolumn{3}{|c|}{$\begin{array}{c}\mathrm{F} 4,36=7.930 \\
(\mathrm{p}<.001)\end{array}$} \\
\hline
\end{tabular}

Tabla 5: Resultados de los análisis de regresión teniendo en cuenta las diferentes metodologías

Analizando los datos de la Tabla 5 se observa lo siguiente. En relación al método de expertos, la edad, la función judicial (en sentido negativo), la forma monárquica, y el ámbito interno explican el $52.3 \%$ de la varianza. En cuanto al estudio compartido, ninguna de las variables de estilos predice el desarrollo de las competencias instrumentales. Por su parte, en el método estudio dirigido, los datos indican que la función 
ejecutiva explica el $12.3 \%$ de la varianza en competencias instrumentales. En relación al aprendizaje basado en problemas, la edad, y la forma monárquica explican el $13.9 \%$ de la varianza. Por último, en cuanto a la metodología estudio de caso, la edad, la función judicial (con signo negativo), la forma monárquica y el ámbito externo (también de forma negativa) explican el 40.9\% de la varianza.

\section{Discusión y conclusiones}

Cada persona tiene unos estilos de pensamiento o una forma de gestionar sus recursos cognitivos predilecta, la cual, en el ámbito académico, se relaciona con su manera de afrontar los procesos de aprendizaje (Zhang, 2002; Zhang, 2004). En consecuencia, el estilo de pensamiento de los alumnos es una variable que puede incidir en su nivel de rendimiento y, por lo tanto, se debe considerar ya que puede mediar en la eficacia de la metodología de enseñanza aprendizaje aplicada (Gokalp, 2013; Riding y Rayner, 2002; Robledo et al., 2010; Tickle, 2001). Desde esta perspectiva, el uso en las aulas universitarias de metodologías de tipo activo con objetivo de promover el desarrollo competencial de los universitarios y dar respuesta a las exigencias del EEES, parece demandar una fase de reflexión e investigación centrada en analizar y valorar realmente la eficacia de estos métodos, tomando en consideración las peculiaridades cognitivas de los estudiantes, como son sus estilos de pensamiento (Santos, Castejón y Martínez, 2014).

En este contexto se ha planteado el desarrollo de este trabajo el cual se ha centrado en valorar el poder predictivo de los estilos de pensamiento de los alumnos en relación a la eficacia de las metodologías activas para promover la adquisición de competencias, previo conocimiento del potencial de cinco métodos activos, aprendizaje basado en problemas, estudio de casos, método de expertos, estudio compartido y estudio dirigido, para contribuir al desarrollo de competencias instrumentales, sistémicas y personales en los universitarios.

En base a los resultados obtenidos en el estudio es posible concluir que todas las metodologías activas contrastadas contribuyen de manera similar a favorecer la asimilación de competencias en los alumnos, confirmándose la hipótesis de investigación en este sentido. Posiblemente el hecho de que las cinco metodologías estudiadas sean de carácter activo y, por lo tanto, aunque tienen aspectos diferenciales que les confieren entidad propia, demanden de manera común una mayor participación y autogestión del aprendizaje por parte del alumno, ha generado que promuevan niveles equitativos de adquisición de competencias. No obstante, sorprende que en todos los métodos analizados las competencias de tipo instrumental han sido las más desarrolladas por el alumnado, seguidas de las de carácter sistémico y, en último lugar, las de tipo personal (Álvarez, Arias, Fidalgo, y Robledo, 2013; Fuente y Justicia, 2003; Robledo, Fidalgo, Arias y Álvarez, 2013). Esta realidad puede estar evidenciando que los métodos docentes denominados activos continúan focalizándose en capacidades de carácter básico o instrumental, de índole cognitivo, metodológico, tecnológico y lingüístico, relegando a un segundo plano las competencias personales relacionadas con la capacidad de utilizar las habilidades comunicativas y críticas, tanto individuales, como sociales. Si bien también es posible que el carácter más estable de las competencias sistémicas y personales conlleve a que sean más difíciles de promover en periodos de tiempo cortos, como el analizado en este estudio. No obstante, estas hipótesis deberían contrastarse en estudios posteriores ya que, de confirmarse, indicarían la necesidad de reajustar las acciones didácticas o los parámetros de algunos enfoques metodológicos.

En cuanto al poder predictivo de los estilos de pensamiento de los estudiantes en relación a la eficacia de las metodologías activas para contribuir al desarrollo de competencias, los análisis se han centrado en las competencias más desarrolladas por los estudiantes (en este caso, las instrumentales). En base a los resultados obtenidos se puede concluir que algunas de las trece variables definitorias de los estilos de aprendizaje influyen sobre el desarrollo de competencias instrumentales en el alumnado universitario, si bien las variables relativas al nivel (global vs. local) no han mostrado tener un papel significativo en este sentido. Además, en la metodología estudio compartido ningún estilo de pensamiento predice el 
desarrollo de competencias instrumentales. Esto evidencia que el estilo de pensamiento de los alumnos no es una variable que desempeñe un papel demasiado relevante para modular la eficacia del método estudio compartido en cuanto a su capacidad para estimular el desarrollo de competencias, posiblemente porque es uno de los métodos que, aun requiriendo la participación activa del alumno, se caracteriza por un alto grado de implicación, control o guía del proceso de enseñanza aprendizaje por parte del profesor, lo cual puede coartar la autonomía del estudiante y limitar su capacidad para actuar de acuerdo a sus maneras predilectas de pensamiento. Y además indica que, en cuanto al desarrollo de competencias mediante el uso de métodos activos, es indiferente que las personas tengan un estilo de pensamiento caracterizado por preferir trabajar con cuestiones amplias o abstractas o prefieran hacerlo de manera más concreta, con detalles específicos.

Atendiendo el resto de variables, los resultados indican que el perfil caracterizado por una función judicial, tendencia conservadora y ámbito externo, se relaciona con menores niveles de competencias instrumentales; mientras que una mayor edad, la función ejecutiva, la forma monárquica y el ámbito interno, se relacionan con un mayor desarrollo de dichas competencias. Adicionalmente, la contribución de cada una de las variables de estilos analizadas, ha demostrado ser diferente dependiendo del método de enseñanza-aprendizaje empleado.

En este caso, es posible concluir, en relación a la dimensión funciones, que la judicial predice negativamente las competencias instrumentales en el método Experto. Es posible que las demandas del método de expertos, consistente en preparar parte de un tema facilitado por el docente para formar a otras personas en el conocimiento del mismo, no sean demasiado compatibles con el estilo judicial, basado en analizar, evaluar información y dar opiniones, y por lo tanto prediga negativamente el desarrollo de las competencias instrumentales. Por su parte, la función ejecutiva tiene una relación positiva con las competencias instrumentales en Estudio Dirigido. Una persona que tiene predilección por este estilo prefiere actuar y realizar tareas y precisamente el método de estudio dirigido demanda este tipo de actuaciones y con ello la puesta en marcha de competencias instrumentales de diversa índole que permitan la realización de las mismas.

Por su parte, el estilo monárquico se relaciona con las competencias instrumentales, en este caso en los métodos Experto, Aprendizaje Basado en Problemas y estudio de caso. Las personas que presentan este estilo de pensamiento tienden a ser decididas en su manera de actuar y a resolver los problemas solventando cualquier obstáculo; siendo precisamente esto lo que deben hacer en los métodos que han resultado significativos, lo cual permite comprender que predigan competencias instrumentales tales como la resolución de problemas, la toma de decisiones o la capacidad de organización y planificación, las cuales posibilitan solventar exitosamente los problemas que se plantean en este tipo de métodos.

En cuanto a la dimensión ámbito, se observa que el interno se relaciona positivamente con las competencias instrumentales en el método Expertos. Las personas con estilo interno tienden a ser introvertidas, centradas en el trabajo y con una pequeña conciencia social, presentando así una forma de pensar o actuar óptimamente compatible con las demandas de las competencias instrumentales. Por su parte, el estilo externo se relaciona negativamente con el desarrollo de competencias en el método de estudio de caso. El estilo de pensamiento externo caracteriza a personas que tienden a ser extrovertidas, centradas en las personas y con una gran conciencia social, de manera que posiblemente las personas con este perfil de pensamiento, al emplear métodos como el estudio de casos, activen más competencias de tipo personal que instrumental, las cuales están menos centradas en las personas y muestran un carácter más técnico que social.

Por lo tanto, a modo de resumen se concluye que diferentes estilos de pensamiento predicen el desarrollo de competencias instrumentales de manera diferente en relación a los distintos métodos activos, lo cual permite confirmar la hipótesis del trabajo evidenciándose cómo aquellos estilos de pensamiento que son más compatibles con las demandas de cada metodología contribuyen a fomentar un mayor desarrollo de competencias (González-Pienda, Roces, Bernardo, y García, 2002). Es decir, al hilo de lo que apoya la 
teoría del autogobierno mental, si los estilos de pensar y por consiguiente las formas de actuar de las personas coinciden con lo que las metodologías demandan de ellas, se pueden obtener resultados óptimos. De manera que cuando las demandas metodológicas son compatibles con los estilos de pensamiento de los alumnos su desarrollo, en este caso competencial, es positivo.

Así pues, desde un punto de vista práctico o aplicado se confirma que el estilo de pensamiento de los alumnos es una de las variables que el profesorado debería considerar a la hora de definir el procedimiento metodológico a implementar en su materia, ya que se trata de una variable relacionada con el rendimiento de los alumnos (Bernardo, Zhang y Callueng, 2002; Bernardo et al., 2009; Cano-García y Hughes, 2000; Zhang, 2002, 2004) que, a su vez, puede modular la eficacia del método y determinar el nivel de desarrollo competencial alcanzado por los estudiantes. Si bien, no hay que olvidar que existen otras variables propias de los alumnos que pueden influir sobre su rendimiento y modular la eficacia metodológica (Albalate, Fageda, y Perdiguero, 2011; Bernardo, et al., 2011; Demirbas y Demirkan, 2007; Robledo et al., 2010); en este estudio en concreto se ha considerado también la edad evidenciándose cómo predictora del desarrollo de competencias en métodos tales como experto, aprendizaje basado en problemas y estudio de caso.

No obstante, estas conclusiones deben tomarse con cierta cautela y considerando las propias limitaciones del estudio. Éstas se relacionan principalmente con el carácter poco representativo de la muestra de alumnos, demasiado pequeña y contextualizada; con el escaso número de docentes implicados en la experiencia, la cual en consecuencia se circunscribe a unas pocas materias y/o titulaciones; y con el tipo de evaluación del desarrollo de competencias realizado, basado en la autopercepción de los propios estudiantes. Por tanto, convendría replicar el estudio con muestras más amplias de estudiantes, implicando a profesorado de diferentes titulaciones, materias y cursos e incluyendo una evaluación de competencias complementaria, de carácter más objetivo.

Sea como sea, parece que los métodos de tipo activo contribuyen en mayor o menor medida a potenciar el desarrollo de competencias, pero que este potencial de la metodología se encuentra mediado a su vez por las propias características internas de los alumnos, en este caso, por sus estilos de pensamiento. Por lo tanto, lo ideal es que los profesores conozcan la forma de pensar y de afrontar el aprendizaje sus alumnos, aspecto complejo que puede ser solventado con la aplicación de cuestionarios empíricamente validados como el aplicado en este trabajo, y a partir de aquí decidan las metodologías didácticas más adecuadas para promover el desarrollo de competencias, sabiendo que la exclusividad en el empleo de un solo método es incompatible con el logro de los diversos objetivos de aprendizaje que se deben alcanzar (Fernández, 2006). Por lo tanto, posiblemente una combinación de varios métodos sea el recurso más adecuado para atender a su vez a la diversidad de perfiles del alumnado, considerando además que los estudiantes pueden tener un perfil de pensamiento combinado o no totalmente definido (Bernardo, Fernández et al., 2011) y que, posiblemente, la estructura organizativa de las universidades no promueva o facilite agrupamientos de alumnos basados en sus similitudes cognitivas, opción que podría resultar también interesante o funcional.

\section{Referencias}

Albalate, D., Fagueda, X., Perdiguero, J. (2011) Éxito académico, características personales y proceso de Bolonia. Revista d'innovació docent universitària, 3, pp. 11-25.

Alcañiz, M., Alemany, R., Bolancé, C., Chuliá, H., Riera, C., Santolino, M. (2013) Importancia de las actitudes y del progreso en competencias sobre el rendimiento académico del estudiante. Revista d'innovació docent universitària, 8 , pp. 20-25.

Allueva, P., Bueno, C. (2011) Estilos de aprendizaje y estilos de pensamiento en estudiantes universitarios. Aprender a aprender y aprender a pensar. Arbor, 187(3), pp. 261-266. 
Álvarez, M., Arias, O., Robledo, P., Fidalgo, R. (2013) Análisis de las competencias transversales en los futuros egresados universitarios de Magisterio. En: O. Arias y R. Fidaldo (Eds.), Innovación Educativa en la Educación Superior Fundamentos, Evaluación e Instrucción (pp. 331-350). Editorial Académica Española, Lap Lambert Academic Publishing GmbH y Co, Madrid.

Arias, O., Fidalgo, R. (2013) Innovación educativa en la Educación Superior. Fundamentos, evaluación e instrucción. Editorial Académica Española, Lap Lambert Academic Publishing GmbH y Co, Madrid.

Bernardo, A., Fernández, E., Cerezo, R., Rodríguez, C., Bernardo, I. (2011) Perfiles de estilos de pensamiento en estudiantes universitarios: implicaciones para el ajuste al Espacio Europeo de Educación Superior. Revista Iberoamericana de Psicología y Salud, 2(2), pp. 145-164.

Bernardo, A., Núñez, J., González-Pienda, J., Rosario, P., Álvarez, L., González, P., Rodríguez, C. (2009) Estilos intelectuales y rendimiento académico: una perspectiva evolutiva. Psicothema, 21, pp. 555-561.

Bernardo, A., Núñez, J.C., Rodríguez, C., Bernardo, I., Fernández, E., Cerezo, R., González, A. (2011) Variables predictoras del rendimiento académico en el EEES: estilos de pensamiento, metas académicas, nota media de entrada a la titulación y horas de estudio. Revista d'innovació docent universitària, 3, pp. 26-34.

Bernardo, A.B., Zhang, L.F., Callueng, C. (2002) Thinking styles and academic achievement among Filipino students. The Journal of Genetic Psychology, 163, pp. 149-163.

Cano-García, F., Hughes, E. (2000) Learning and thinking styles: An analysis of their interrelationship and influence on academic achievement. Educational Psychology, 20, pp. 413-430.

De Miguel, M. (2005) Modalidades de enseñanza centradas en el desarrollo de competencias. Orientaciones para promover el cambio metodológico en el Espacio Europeo de Educación Superior. Ediciones de la Universidad de Oviedo, Oviedo.

Demirbas, O., Demirkan, H. (2007) Learning styles of design students and the relationship of academic performance and gender in design education. Learning and Instruction, 17, pp. 345-359.

Estrategia Universidad 2015 (2015) Universidades para el progreso, el bienestar y la competitividad. Ministerio de Ciencia e Innovación, Gobierno de España, Madrid.

Fer, S. (2007) What are the thinking styles of turkishstudent teachers? Teachers college record, 109(6), pp. 14881516.

Fernández, A. (2006) Metodologías activas para la formación de competencias. Educatio Siglo XXI, 24, pp. 35-56.

Fidalgo, R., Arias, O., García, J.N. (2007) Una aproximación empírica a la evaluación de las competencias transversales en el grado de magisterio. Artículo presentado a I Jornada Internacional UPM sobre Innovación Educativa y Convergencia Europea, Madrid.

Fuente, J., Justicia, F. (2003) Regulación de la enseñanza para la autorregulación del aprendizaje en la universidad. Aula Abierta, 82, pp. 161-171.

Gokalp, M. (2013) The effect of students' learning styles to their academic success. Creative Education, 4, pp. 627632.

González, V. (2002) ¿Qué significa ser un profesional competente? Reflexiones desde una perspectiva psicológica. Revista Iberoamericana de educación, 22, pp. 45-53.

González-Pienda, J.A., Roces, C., Bernardo, A., García, M.S. (2002) Estilos de aprendizaje y estilos de pensamiento. En: J.A. González-Pienda, R.G. Cabanach, J.C. Núñez, A. Valle (Eds.), Manual de psicología de la educación (pp. 165-186). Pirámide, Madrid. 
Gridley, M. (2007) Differences in thinking styles of artists and engineers. The Career Development Quarterly, 56, pp. 177-182.

Martínez, M., Crespo, E. (2008) Una aproximación a los factores que influyen en la motivación del alumnado de filología inglesa y traducción e interpretación. VI Jornadas de redes de investigación en docencia universitaria. Alicante, España.

Mogollón, O., Garrido, E. (2004) Estilos de pensamiento de los estudiantes de la Universidad Pública de Navarra y de la Universidad de Pamplona, Colombia. Huarte de San Juan psicología y pedagogía, 11, pp. 87-120.

Núñez, J.C., González-Pienda, J.A., Bernardo, A., Álvarez, L., Rosário, P., Valle, A., et al. (2008) Intellectual abilities, thinking styles and academic achievement. En: A. Valle, J.C. Núñez, R.G. Cabanach, J.A. GonzálezPienda, y S. Rodríguez (Eds.), Handbook of instructional resources and their applications in the classroom (pp. 4566). Nova Science, Nueva York.

Riding, R., Rayner, S. (2002) Cognitive Styles and Learning Strategies. London: David Fulton Publishers.

Robledo, P., Fidalgo, R., Arias, O., Álvarez, M. (2013) Estudio comparativo de los enfoques tradicionales frente a los innovadores en la enseñanza universitaria. En: O. Arias y R. Fidaldo (Eds.), Innovación Educativa en la Educación Superior Fundamentos, Evaluación e Instrucción (pp. 313-330). Editorial Académica Española, Lap Lambert Academic Publishing GmbH y Co, Madrid.

Robledo, P., García, J., Díez, C., Álvarez, L., Marbán, J., de Caso, A., et al. (2010) Estilos de pensamiento y aprendizaje en estudiantes de magisterio y psicopedagogía: diferencias según curso y especialidad. Escritos de Psicología, 3(3), pp. 27-36.

Santos, L., Castejón, J., Martínez, F. (2014) Análisis y propuestas de cambio en la metodología y la evaluación en el Espacio Europeo de Educación Superior. Revista Iberoamericana de Evaluación Educativa, 7(3), pp. 127-144.

Sternberg, R.J. (1988) Mental self-government: A theory of intellectual styles and their development. Human Development, 31, pp. 197-224.

Sternberg, R.J. (1994) Allowing for thinking styles. Educational Leadership, 52, pp. 36-39.

Sternberg, R.J. (1997) Thinking styles. Cambridge, UK: University of Cambridge.

Sternberg, R.J. (1999) Estilos de pensamiento: Claves para identificar nuestro modo de pensar y enriquecer nuestra capacidad de reflexión. Paidós, Barcelona.

Sternberg, R.J., Wagner, R.K. (1991) MSG Thinking Styles Inventory (manual). Departamento de Psicología, Universidad de Yale. Adaptación de J. A. González Pienda, J.C Núñez, S. González Pumariega, L. Álvarez, C. Roces, y M. S. García (1997). Universidad de Oviedo.

Sternberg, R.J., Grigorenko, E.L. (1995) Styles of thinking in the school. European Journal for High Ability, 6, pp. 201-219.

Tickle, S. (2001) What have we learnt about student learning? A review of the research on study approach and style. Kybernetes, 30, pp. 955-969.

Villardón, L. (coord.) (2015) Competencias genéricas en educación superior. Metodologías específicas para su desarrollo. Narcea, Madrid.

Zhang, L.F. (2002) Thinking styles: Their relationships with modes of thinking and academic performance. Educational Psychology, 22, pp. 331-348.

Zhang, L.F. (2004) Revisiting the predictive power of thinking styles for academic performance. The Journal of Psychology, 138, pp. 351-370. 